\title{
miR-221-3p/222-3p Cluster Expression in Human Adipose Tis- sue is Related to Obesity and Type 2 Diabetes
}

\author{
Adriana-Mariel Gentile ${ }^{1 *}$, Said Lhamyani ${ }^{*}$, Mercedes Clemente Postigo ${ }^{2,3}$, Enrique Estepa1, Maria Mengual-Mesa ${ }^{1}$, \\ Francisco-Javier Bermúdez-Silvaa, ${ }^{1,4}$ Alberto Rodriguez Cañete ${ }^{5}$,Francisco J Tinahones, ${ }^{1,3,6}$, Gabriel Olveira ${ }^{1,4}$ and \\ Rajaa El Bekay ${ }^{1,3^{* *}}$
}

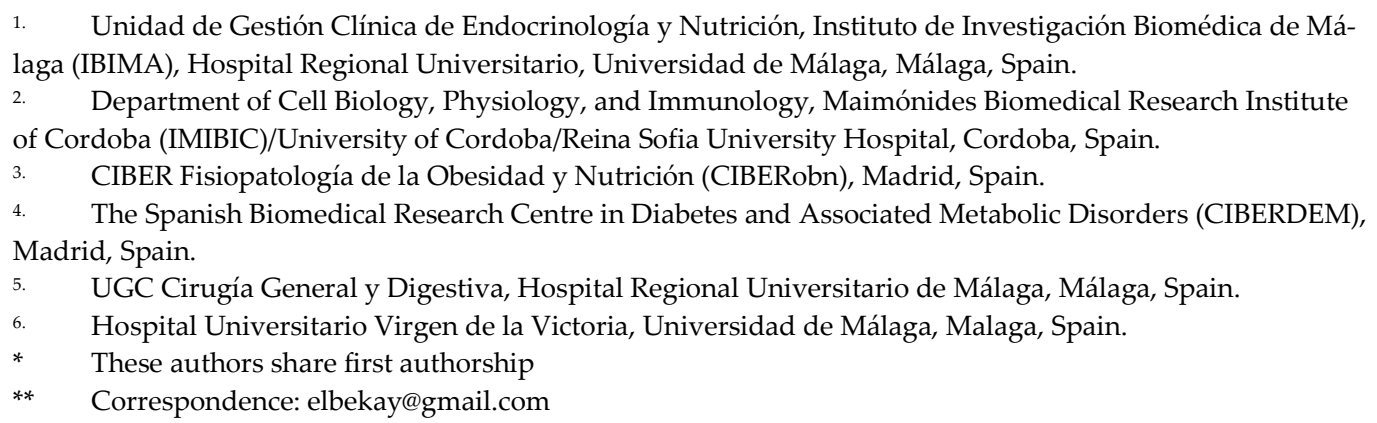

\begin{abstract}
Background: The course of obesity and type 2 diabetes (T2D) development is highly dependent on adipose tissue (AT) angiogenesis. Moreover, angiogenic microRNAs (miRNAs) play pivotal role in AT functionality. The aim of this study was to analyze the relationship of the human AT miR-221-3p/222-3p cluster and their regulatory network with obesity and T2D. Methods: miR221-3p/222-3p and their target genes (TG) expression levels were measured in visceral and subcutaneous ATs from patients classified according to their BMI and to their glycemic status with a high degree of insulin resistance (IR) and T2D. In silico analyses of miR-221-3p/222-3p and their TGs were performed to identify relevant signaling pathways. Results: A multivariate analysis, including the simultaneous expression of miR-221-3p and miR-222-3p as dependent variables, showed significant differences considering the variables; tissue depot, obesity, IR and T2D altogether as independent variables. In addition, miRNAs and their TGs were differentially expressed according to obesity degree, glycemic status, and AT depot type. Our in silico analysis showed that miR-221-3p/222-3p cluster TGs are mostly involved in angiogenesis, WNT signaling pathway and apoptosis. Conclusion: These findings suggest that the miR-221-3p/222-3p cluster and their related regulatory networks could represent tangible targets for the management of obesity and associated metabolic disorders.
\end{abstract}

Keywords: miR-221-3p/222-3p cluster; human adipose tissue; obesity; type 2 diabetes

\section{Introduction}

Obesity has increased considerably in recent decades and is a huge public health problem [1]. Obesity is generally accompanied by other metabolic diseases, such as type 2 diabetes (T2D), insulin resistance (IR), fatty liver and cardiovascular diseases. Dysfunctional adipose tissue (AT) has extensively been involved in the development of these obesity-associated comorbidities [2,3].

AT is a highly dynamic endocrine tissue contributing to metabolic homeostasis [4]. The role of AT as energy storage is crucial for maintaining lipid homeostasis and avoiding ectopic fat accumulation [5]. Nonetheless, when it becomes severely dysfunctional and expands inappropriately to store the energy excess, AT leads to metabolic alterations associated with obesity such as insulin resistance and T2D [6,7]. This dysfunction is due to its vascularization (angiogenesis) decrease, hypoxia increase, and inflammation [8,9]. 
Thus, angiogenesis is considered a key process for healthy AT expansion under chronic overnutrition conditions which prevents hypoxia and allows for the correct supply of nutrients to cells $[10,11]$. In this regard, it has been proposed that the onset of AT dysfunction is essentially determined by its capacity to store excess energy, rather than by its size. Thus, there is an inter-individual variability in AT expandability and remodeling which determines whether the metabolically healthy status is maintained or not [12]. This would explain the existence of metabolically healthy obese subjects and lean subjects with metabolic disease [10,12-15].

microRNAs (miRNAs), small non-coding RNAs that regulate gene expression, have recently gained interest due to their role in the development of fat cells as well as in obesity and metabolic disorders [16]. Several studies have shown that the expression of miRNAs in preadipocytes (miR-221, miR-125b, miR-34a, miR-100, miR-130b, miR-210 and miR-185) is altered in obesity [17]. Also, our group has recently reported the dysregulation of several AT miRNAs in T2D and obesity, including miR-20b, miR-296, and Let-7f [18], and the involvement of others (miR21) in AT functionality regulation [19].

miR-221-3p and miR-222-3p are well described to be primarily involved in angiogenesis by controlling multiple angiogenic genes [12]. These miRNAs have been related to metabolic diseases in AT and as biomarkers [12, 20-22]. In addition, miR-221 and miR-222 have been described to be novel diagnostic, prognostic and therapeutic biomarkers in various diseases including cancer and inflammatory diseases [23] and have been found to be upregulated in insulin resistance and certain types of cancer [24].

It has also been proposed that a complex miRNA regulatory network may be controlling AT functionality, and that miRNA expression profile alteration could be contributing to AT dysfunction related to T2D [24]. However, the mechanisms underlying the involvement of these miRNAs in AT regulation remain to be well determined. Taking into consideration the relevant role that the miR-221-3p/222-3p cluster can have in maintaining AT homeostasis, the present work aims is to analyze the relationship of this cluster and its target gene network in human AT with obesity and T2D

\section{Materials and Methods}

\subsection{Patients, study design and AT collection}

The study included obese patients with a body mass index $(\mathrm{BMI})=30-40 \mathrm{Kg} / \mathrm{m}^{2}$ and normal-weight subjects $\left(\mathrm{BMI}=20-25 \mathrm{Kg} / \mathrm{m}^{2}\right)$ who underwent laparoscopic surgery for hiatus hernia or cholelithiasis $\left(\mathrm{BMI}<40 \mathrm{Kg} / \mathrm{m}^{2}\right)$ at the Virgen de la Victoria University Hospital (Málaga, Spain).

The criteria to assign participants to the different groups were as follows: normoglycemic (NG) with blood glucose levels after at least 8 hours of fasting $\leq 110 \mathrm{mg} / \mathrm{dl}$ and with homeostatic model assessment for IR (HOMA-IR) $\leq 3.5$ ); IR those with glycemia $\leq 110$ $\mathrm{mg} / \mathrm{dl}$ and HOMA-IR> 4 and T2D with glycemia $>110 \mathrm{mg} / \mathrm{dl}$ and HOMA-IR $>4$. Then, participants were classified into five groups according to their BMI, HOMA-IR, and glycemic status in NG-normoweight subjects (NG-NW), NG-obese subjects (NG-OB), NGobese subjects with IR (IR-OB), T2D-normoweight subjects (D-NW) and T2D-obese subjects (D-OB). The exclusion criteria were, patients who had insulin treatment for T2D, prior cardiovascular disease, acute or chronic inflammatory disease or infectious disease, patients who refused to participate in the study and patients who had a HOMA-IR between 3.5-4.

Both SAT and VAT tissue samples were obtained during the surgical procedure from abdominal and omental regions, respectively. Biopsies were washed in physiological saline solution, promptly frozen in liquid nitrogen and stored at $-80^{\circ} \mathrm{C}$ until assays. All participants provided written informed consent and the study was reviewed and approved by the Ethics and Research Committee of the Virgen de la Victoria University Hospital (Málaga, Spain).

\subsection{Laboratory analysis}


After an overnight fast and before surgery, blood samples were obtained from human subjects from the antecubital vein and placed in vacutainer tubes (BD vacutainer ${ }^{\mathrm{TM}}$ ). The serum was separated by centrifugation for $10 \mathrm{~min}$ at $4000 \mathrm{rpm}$ and immediately frozen at $-80^{\circ} \mathrm{C}$ until analysis. Serum glucose, cholesterol, triglycerides, and HDL-cholesterol levels were measured in a Dimension auto-analyzer (Dade Behring Inc., Illinois, USA) by enzymatic methods (Randox Laboratories Ltd., County Antrim, UK). LDL-cholesterol was calculated with the Friedewald equation. Insulin was quantified by radioimmunoassay (BioSource International, California, USA) and IR was calculated using HOMA-IR as previously described [25].

\section{3. miRNA extraction and real-time quantitative PCR ( $q P C R$ )}

miRNAs from AT were isolated as previously described [18] using mirVana ${ }^{\mathrm{TM}}$ miRNA Isolation Kit (Ambion life technologies, Carlsbad, CA, USA), according to the manufacturer's guidelines. miRNA concentration and purity were determined using a NanoDrop1000 spectrophotometer (Thermo Fischer Scientific, Inc., MA, USA). cDNA was obtained using the TaqMan ${ }^{\circledR}$ MicroRNA Reverse Transcription Kit (Applied Biosystems, CA, USA) and specific primers and probes for each miRNA were used (TaqMan ${ }^{\circledR} \mathrm{Mi}^{-}$ croRNA Assay, Applied Biosystems): has-miR-221-3p (assay ID 000524); hsa-miR-222-3p (assay ID 002276). hsa-miR-16 (TaqMan ${ }^{\circledR}$ MicroRNA Assay ID 000391; Applied Biosystems) was assessed using the Bestkeeper software to determine their usability as reference gene (http://www.gene-quantification.de/bestkeeper.html) and used as the endogenous control. A constant amount of $5 \mathrm{ng}$ of miRNA was used to perform reverse transcription in a mix containing $5 \mu \mathrm{l}$ RNA, $7 \mu \mathrm{l}$ RT-MasterMix and $3 \mu \mathrm{l}$ RT-primers. The reverse transcription program consisted of $30 \mathrm{~min}$ at $16^{\circ} \mathrm{C}$, $30 \mathrm{~min}$ at $42^{\circ} \mathrm{C}$, and $5 \mathrm{~min}$ at $85^{\circ} \mathrm{C}$. miRNA expression levels were assessed by real-time qPCR using Applied Biosystems 7500 Fast Real-Time PCR System (Applied Biosystems, CA, USA). Each sample was assessed in duplicate and relative quantification of miRNA levels was performed by the comparative threshold cycle $(\mathrm{Ct})$ method according to the manufacturer's guidelines.

\section{4. $m R N A$ isolation and $q P C R$}

The RNeasy ${ }^{\circledR}$ Lipid Tissue Mini Kit (Qiagen, Washington, MD, USA) was used to isolate total mRNA from AT [18]. RNA concentration and purity were determined using a NanoDrop1000 spectrophotometer (Thermo Fischer Scientific, Inc.,). cDNA synthesis was obtained with Transcriptor Reverse Transcriptase Kit (Roche Diagnostic, Barcelona, Spain) according to the manufacturer's instructions and the real-time qPCR performed with TaqMan ${ }^{\mathrm{TM}}$ Fast Advanced Master Mix (Applied biosystems. Thermo Fisher Scientific. Seville, Spain) in a StepOne ${ }^{\mathrm{TM}}$ Real-Time PCR System (Applied Biosystems).

The reference gene (Cyclophilin A) was selected using Bestkeeper software (http://www.gene-quantification.de/bestkeeper.html). Probes used for mRNA detection are detailed in Table S1. Each sample was assessed in duplicate and relative quantification of mRNA levels was performed by the formula $2^{-\Delta \mathrm{Ct}}$ according to the manufacturer's guidelines.

\subsection{Bioinformatic analysis}

The miRTarBase 4.0 website (http://mirtarbase.mbc.nctu.edu.tw/) was used for identifying previously validated TG of miR-221-3p and miR-222-3p. Moreover, the miRWalk 2.0 (http://www.umm.uni-heidelberg.de/apps/zmf/mirwalk/index.html) database was also used for identifying predicted miR-221-3p and miR-222-3p TGs involved in angiogenesis, adipogenesis and apoptosis pathways. The PANTHER Database (Protein ANalysis Through Evolutionary Relationship) Classification System (http://www.pantherdb.org/) was applied to annotate the signaling pathways of validated target genes. GeneCodis3 (http://genecodis.dacya.ucm.es) was applied for enrichment analysis of validated and non-validated target genes. 
The interactions among miRNAs, biological processes and the differentially expressed TGs were visualized with Cytoscape v.3.2.1 software (http://www.cytoscape.org/).

The binding sites of miR-221-3p and miR-222-3p within the selected TGs were predicted using TargetScan Human 7.2 which indicates the conservation force (according to the position of the seed region), the evolutionary conservation, and binding efficiency.

\subsection{Statistical analysis}

The results are expressed as the mean \pm SEM. We tested the normality of the distribution of continuous variables using Shapiro-Wilk statistics. Data were analyzed by Mann Whitney U-test or Student's t-test for non-parametric or for parametric data, respectively. Multivariate general linear and regression models were used for data analysis as follows: (a) The obesity, T2D, type of AT depot and different patients' groups were introduced as independent. (b) The expression levels of miR-221-3p and miR-222-3p or TGs were introduced as dependent variables. Associations between age and miRNA expression levels were analyzed using Pearson's correlation. Statistical analyses were carried out with the statistical software package SPSS (version 22.0; SPSS Inc., Chicago, IL, USA). Pvalue $<0.05$ was considered statistically significant.

\section{Results}

\subsection{Patient characteristics}

The anthropometric and clinical variables of the study subjects are summarized in Table 1.

We found differences in age between diabetic and normoglycemic lean patients. However, Bivariate correlation analyses did not show significant differences between miR-221-3p $(\mathrm{R}=0.054 ; p=0.800)$ or miR-222-3p $(\mathrm{R}=0.161 ; p=0.453)$ expression levels in VAT or SAT and the patient's age.

\subsection{Human miR-221-3p and miR-222-3p expression profiles and their association with obesity,} T2D and AT depot

A general linear multivariate model, including the interception, was performed among miR-221-3p and miR-222-3p expression levels as dependent variables, and tissue depot, obesity degree and glycemic status as independent variables. Thus, it was able to show the discriminative power of variables. These differences are expressed by the interception of the model (Wilks' $\lambda=0.301, \mathrm{~F}=17.425, \mathrm{p}<0.0001$ ). Specifically, a tissue depot effect was observed ( $p=0.036$ for miR-221-3p and $p=0.019$ for miR-222-3p) and obesity effect $(p=0.024$ for miR-221-3p and $p=0,057$ for miR-222-3p). In addition, an interaction effect between obesity and tissue depot was observed ( $p=0,041$ for miR-221), particularly between healthy obese $(p=0.043)$ and diabetic obese $(p=0.049)$ subjects when compared with healthy normoweight subjects.

To further analyze the impact of obesity, type of tissue and T2D on the expression of miRNAs, linear regression models were used. We included the expressions of each miRNA as dependent variables in separate models, and obesity, type of tissue and T2D as independent variables. The regression model for miR-221-3p $\left(R^{2}=0.541 ; F=5.215 ; p=0.036\right)$ suggested that $54 \%$ of the variation in the expression of miRNA-221 could be explained by the positive effect of obesity $(\beta=0.421 ; p=0.032)$ and by the negative effect of tissue $(\beta=$ -0.387; $p=0.047$ ) (Figure1). The regression model for miR-222-3p (R2=0.477; F=6.841; $\mathrm{p}=0.019)$ suggested that $48 \%$ of the variation in miRNA-222-3p expression may be explained by the negative effect of tissue $(\beta=-0.473 ; p=0.015)$ (Figure1). These results indicate that, in the presence of both obesity and T2D, the expression levels of miR-221-3p and miR-222-3p are tissue-dependent (Figure 1).

\subsection{In silico analysis of miRNA-221-3p/222-3p TGs}


The in silico analysis with miRTarBase highlighted 911 validated TGs for miR-221-3p and miR-222-3p, of which, 53 were common TGs for these two miRNAs (Table 2 and Table S2). Signaling pathways analysis with PANTHER showed that miR-221-3p and miR222-3p TGs are mainly involved in angiogenesis, apoptosis, inflammation, adipogenesis, PDGF, interleukin and the Wnt signaling pathways (Figure 2A). Moreover, analysis with miRWalk showed that 17 predicted TGs for the mature sequences of both miR-221-3p and miR-222-3p are involved in angiogenesis, apoptosis and adipogenesis (Table 2 and Table $\mathrm{S} 2)$.

\subsection{Annotation enrichment analysis of miR-221-3p/222-3p TGs}

GeneCodis3 was used for enrichment analysis. A total of 24 TGs (Validated:7; predicted:17) were highlighted and three of them (DVL2, ETS, IL1rap), related to angiogenesis and apoptosis signaling pathways, have the best score and specificity (Figure 2B). Figure $2 \mathrm{C}$ shows how these TGs could be interacting in these pathways.

\subsection{Identification of binding sites of miRNAs within TGs}

Most miRNA bind to mRNA through canonical sites, but some miRNAs bind through non-canonical sites. However, they are not equally effective [26]. The TargetScan Human tool allows for the detection of binding sites, with a precision of site-recognition predictions that was reported to be comparable to in vivo approaches [26]. The value of the context++ score percentile measures the effectiveness of each binding site. In addition, the biological relevance of predicted miRNA-target interactions, is measured through the probability of conserved targeting (PCT) [27]. The PCT value has a range between 0 and 1 (less or more conserved, respectively, and conserved sites are more likely to have detectable biological functions [27]. Our results showed that miR-221-3p has effective binding sites within ETS1, DVL2 and IL1RAP (context++ score percentile: 70\%, 65\% and $94 \%$, respectively) as well as miR-222-3p (context++ score percentile: $72 \%, 70 \%$ and $94 \%$, respectively). Furthermore, the PCT value that indicates the probability of segmentation being conserved for a single target site [27] showing a value of 0.10 , which indicates evolutionary conservation (Table S3).

3.6. ETS1, DVL2 and IL1RAP gene expression in human VAT and SAT according to the obesity degree and IR and the glycemic status

A general linear multivariate model, including the interception, was performed among expression levels of DVL2, ETS1 and IL1RAP as dependent variables, and tissue depot, obesity degree and IR, and glycemic status as independent variables. Thus, it was able to show the discriminative power of variables. These differences are expressed by the interception of the model (Wilks' $\lambda=0.301, F=48.417, p<0.0001$ ), tissue $\operatorname{depot}($ Wilks' $\lambda=0.484$, $\mathrm{F}=7.814, p=0.001$ ), obesity degree (Wilks' $\lambda=0.465, \mathrm{~F}=8.440, p=0.001$ ) and IR degree (Wilks' $\lambda$ $=0.261, \mathrm{~F}=20.747, p=0.0001)$. Specifically, a tissue depot effect was observed for IL1RAP $(p=0.004)$, an obesity degree effect for DVL2 ( $\mathrm{p}=0.0001)$ and ETS1 (0.026), an IR degree effect for DVL2 ( $p=0.0001)$ and ETS1 (0.001), an interaction effect between tissue depot and obesity degree for DVL2 $(p=0.001)$ and an interaction effect between tissue depot and IR degree for DVL2 ( $\mathrm{p}=0.0001)$ and ETS1 ( $p=0.003)$ (Figure 3).

To further analyze the impact of obesity degree and IR, type of tissue and glycemic status in the expression of TGs we used linear regression models. We included the expressions of each TG as dependent variables in separate models, and obesity degree and IR, AT type, and glycemic status as independent variables. The first regression model $\left(\mathrm{R}^{2}=\right.$ $0.459 ; p=0.002$ ) demonstrated that $46 \%$ of the variation in the expression of DVL2 could be explained by the positive effect of obesity $(\beta=0.513 ; p=0.006)$ and by the negative effect of $\operatorname{IR}(\beta=-0.829 ; p=0.0001)$. The second regression model $\left(R^{2}=0.321 ; p=0.029\right)$ suggested that $32 \%$ of the variation in the expression of ETS1 could be explained by the negative effect of $\operatorname{IR}(\beta=-0.738 ; p=0.003)$. The third regression model $\left(R^{2}=0.364 ; p=0.013\right)$ suggested that $36 \%$ 
of the variation in the expression of IL1RAP could be explained by the negative effect of tissue $(\beta=-0.590 ; p=0.0001) \quad$ (Figure 3A).

Figure 3B summarizes the potential relationships between ETS1, DVL2, IL1rap, miR$221-3 p / 222-3 p$ and the biological processes in which they are involved. The interaction network suggested that the miR-221-3p/222-3p cluster could coordinately regulate angiogenesis, apoptosis and Wnt signaling pathway through the modulation of their common TGs, such as ETS1, DVL2 and IL1rap

\subsection{Expression and Regulatory Patterns of miRNAs/mRNAs}

Figure 4 shows that, in the presence of both obesity and T2D factors, the expression levels of both members of the miR-221-3p/222-3p cluster showed a similar pattern of regulation miRNA/mRNA in both VAT and SAT (Figure 4). Thus, in VAT, an under-expression of the miR-221-3p / 222-3p cluster was related to an up-regulation of ETS1, DVL2 and Il1RAp, whilst in SAT, an overexpression of the cluster was related to downregulation of these three genes.

\section{Discussion}

Our results showed that the expression levels of miR-221-3p and miR-222-3p are tissue-dependent and they are related to both obesity and T2D. Consistent with our findings, miR-221-3p has been shown to be involved in promoting AT inflammation [28.29] and, together with miR-222-3p, contributes to cardiovascular pathology through their effects on fat and glucose metabolism [30] and is related to fat depots, obesity, IR and T2D [20]. Although our data conforms with these previous findings, our study provides novel evidence that miR-221-3p/222-3p cluster expression in human AT could be related to obesity and fat depot when IR and T2D were considered. To the best of our knowledge, this is the first study that describes a possible connection between obesity, IR, T2D and the miR221-3p/222-3p cluster expression in human VAT and SAT. This relation could be mediated through some signaling pathways involved in AT functionality regulation.

Our bioinformatic study highlighted 24 TGs common for both miR-221-3p and miR222-3p. Three of them; E26 transformation specific-1 (ETS1), Dishevelled 2 (DVL2) and Interleukin 1 Receptor Accessory Protein (IL1RAP) were described to play a pivotal role in regulating AT functionality through angiogenesis, apoptosis, and Wnt signaling pathways control.

DVL is a cytoplasmic adaptor protein necessary for Wnt signaling and functions in canonical signaling branches to regulate cell proliferation and cell fate decision. Whereas noncanonical Wnt signaling controls cell polarity, cell migration and some other events related to the Wnt signaling pathway [31].

ETS1 is the older member of the ETS transcriptional factor family known to be involved in cellular differentiation, tissue remodeling, angiogenesis, drug resistance and tumorigenesis. Although usually regarded as an oncogene, its apoptosis-promoting activity has also been reported [32].

The IL-1 family is a group of cytokines that play a central role in the regulation of immune and inflammatory responses [33]. Interleukin-1 is a key inflammatory cytokine that mediates its effects through a type I receptor and a receptor accessory protein. These two molecules are members of a wider family of proteins that have in common the presence of immunoglobulin domains in the extracellular region of the protein and a TIR domain in the cytoplasmic region [34].

The three TGs of these miRNAs showed differential expression profiles depending on obesity, IR and AT depots when glycemic status was considered.

miR-221-3p and miR-222-3p have been shown to be involved in the regulation of apoptosis, WNT signaling pathway and angiogenesis by regulating several TGs [35-38]. Specifically, ETS1 and DVL2 are known to be key regulators of the WNT signaling pathway and angiogenesis $[39,40]$, whilst IL1RAP is described as key regulators of apoptosis [41]. 
In a metabolic disease such as T2D IR, IL1RAP has been described to be involved in the macrophage-mediated chronic inflammatory response [33]. In AT, the inflammatory response is mostly mediated by infiltrated macrophages that play a relevant role in controlling its functionality [42] and thus we consider that, in human AT, IL1RAP would play a relevant role in macrophages-mediated inflammatory processes regulation.

In the present study, even if both miR221 and miR-222 are known to usually perform their regulation jointly, as they are part of the same cluster, we analyzed their expression levels in relation to obesity and fat depot, considering at the same time the three variables: obesity, T2D, and AT depot. Thus, the multivariate analysis highlights that $54 \%$ and $47 \%$ of expression level of each one, respectively, are affected positively by obesity and negatively by the type of tissue. These data confirm that the involvement of miR221/miR-222 cluster in AT regulation should be studied considering the interaction of the two miRNAs (miR221 and miR-222) within tissue. In this regard, Kabekkodu has reported [43], that members of a miRNA group present different expression levels and co-expression patterns indicating the possible existence of miRNA-miRNA interactions between groups and intra-groups, and that the expression of each miRNA within the same group depends on the expression of other cluster members.

Moreover, we here describe that in human AT DVL2 expression level is fat depot and obesity dependent, which is in line with other previous data relating this gene to insulin sensibility [44], and we provide new data pointing to the fact that ETS2 and IL1RAP expression levels seem to be also related to fat depot and IR. Altogether, our data suggest that the miR-221-3p/222-3p cluster could be regulating AT functionality through DVL2, ETS1 and IL1RAP regulation during obesity and T2D.

Finally, it is important to highlight that the most novel research of this study is applying multivariate analysis to investigate the interactions among the both ATs, VAT and SAT, the miR-221-3p/222-3p cluster and to identify their common potential TGs in silico. We were able to prioritize three common TGs (ETS1, DVL2 and IL1RAP), which showed significantly different expression profiles in both VAT and SAT in patients with obesity and T2D. Furthermore, the binding site prediction analysis provided additional valuable data that confirm direct regulation of miR-221-3p and miR-222-3p into gene-regulatory networks of ETS1, DVL2 and IL1RAP concerning to obesity and T2D.

\section{Conclusion}

The relationship among obesity, T2D, miR-221-3p/222-3p cluster and their TGs (ETS1, DVL2 and IL1RAP) represent a promising line of future research to determine the role of this network in AT functionality regulation in both T2D and obesity. These findings are expected to serve as a platform for drug development for both diseases.

Author Contributions: AMG and SL; experimental, bioinformatics and statistical analysis and data interpretations, MCP, MMM, EE and FJBS; data analysis and manuscript edition, ARC, GO and FT; patients recruitment, surgery and clinical analysis, AMG; writing original draft, RE; writing-review and editing, visualization, supervision, project administration, funding acquisition.

Funding: This work was supported in part by grants from the Instituto de Salud Carlos III (ISCIII)/FEDER-UE (PI18/00785), from Consejería de Transformación Económica, Industria, Conocimiento y Universidades Dirección General de Investigación y Transferencia del Conocimiento (PI20-01274) co-funded by Fondo Europeo de Desarrollo Regional-FEDER. S.L. is a recipient of a post-doctoral grant Plan Andaluz de Investigación, Desarrollo e Innovación (DOC-01138) from Consejería de Economía, Conocimiento, Empresas y Universidades. R.E.B. and FJBS are under a contract from the 'Nicolas Monarde' (C-0030-2016, RC-0005-2016) program from the Servicio Andaluz de Salud, Regional Ministry of Health of the Andalusian Government, Andalusia. Spain. M.C.P. was a recipient of a post-doctoral grant Juan de la Cierva Formación (FJCI-2017-32194) from the Ministerio de Ciencia, Innovación y Universidades (Spain).

Data Availability Statement: Not applicable.

Acknowledgments: The authors wish to thank all the subjects for their collaboration. Centro de Investigación Biomédica en Red de Fisiopatología de la Obesidad y Nutrición (CIBERobn) and 
Centro de Investigación Biomédica en Red de Diabetes y Enfermedades Metabólicas Asociadas. (CIBERdem) are part of the 'Instituto de Salud del Carlos III' (ISCIII).

Conflicts of Interest: The authors have no conflicts of interests to declare.

\section{References}

1. Chooi, Y.C.; Ding, C.; Magkos, F. The epidemiology of obesity. Metabolism. 2019; 92:6-10.

2. Longo, M.; Zatterale, F.; Naderi, J.; Parrillo, L.; Formisano, P.; Raciti, G. A.; Beguinot, F. and Miele, M. Adipose Tissue Dysfunction as Determinant of Obesity-Associated Metabolic Complications. Int J Mol Sci. 554 2019;20(9):2358.

3. Ferrara, D.; Montecucco, F.; Dallegri, F.; Carbone, F. Impact of different ectopic fat depots on cardiovascular and metabolic diseases. J Cell Physiol. 2019;234(12):21630-21641.

4. Barchetta, I.; Cimini, F.A.; Ciccarelli, G.; Baroni, M.G.; Cavallo, M.G.; Sick fat: the good and the bad of old and new circulating markers of adipose tissue inflammation. J Endocrinol Invest. 2019;42(11):1257-1272.

5. Smith, U. Abdominal obesity: a marker of ectopic fat accumulation. J Clin Invest. 2015;125(5):1790-2.

6. Blüher, M. Adipose tissue dysfunction in obesity. Exp Clin Endocrinol Diabetes. 2009; 117(6):241-50.

7. Oliva-Olivera, W.; Coín-Aragüez, L.; Lhamyani, S.; Clemente-Postigo, M.; Torres, J.A.; Bernal-López, M.R.; El Bekay, R.; Tinahones, F.J. Adipogenic Impairment of Adipose Tissue-Derived Mesenchymal Stem Cells in Subjects With Metabolic Syndrome: Possible Protective Role of FGF2. J Clin Endocrinol Metab. 2017; 564 102(2):478-487.

8. Manna, P.; Jain, S.K.Obesity, Oxidative Stress, Adipose Tissue Dysfunction, and the Associated Health Risks: Causes and Therapeutic Strategies. Metab Syndr Relat Disord. 2015; 13(10):423-44.

9. Jung U.J, Choi M.S. Obesity and its metabolic complications: the role of adipokines and the relationship 568 between obesity, inflammation, insulin resistance, dyslipidemia and nonalcoholic fatty liver disease. Int J Mol Sci. 2014 11;15(4):6184-223.

10. Cao Y. Angiogenesis modulates adipogenesis and obesity. J Clin Invest. 2007 117(9):2362-8.

11. Crewe, C.; An, Y.A.; Scherer, P.E. The ominous triad of adipose tissue dysfunction: inflammation, fibrosis, and impaired angiogenesis. J Clin Invest. 2017;127(1):74-82.

12. Di Stefano, A.B.; Massihnia, D.; Grisafi, F.; Castiglia, M.; Toia, F.; Montesano, L.; Russo, A.; Moschella, F.; Cordova, A. Adipose tissue, angiogenesis and angio-MIR under physiological and pathological conditions. Eur J Cell Biol. 2019; 98(2-4):53-64

13. Bråkenhielm E., Cao R., Gao B., Angelin B., Cannon B., Parini P, Cao Y. Angiogenesis inhibitor, TNP-470, prevents diet-induced and genetic obesity in mice. Circ Res. 2004. 25;94(12):1579-88

14. Neels J.G, Thinnes T, Loskutoff D.J. Angiogenesis in an in vivo model of adipose tissue development. FASEB J. 2004 18(9):9835.

15. Rupnick M.A, Panigrahy D., Zhang C.Y, Dallabrida S.M, Lowell B.B, Langer R, Folkman M.J. Adipose tissue mass can be regulated through the vasculature. Proc Natl Acad Sci U S A. 2002 6;99(16):10730-5.

16. Arner, P.; Kulyté, A. MicroRNA regulatory networks in human adipose tissue and obesity. Nat Rev 596 Endocrinol. 2015;11(5):276-88. 597

17. Ortega, F.J.; Moreno-Navarrete, J.M.; Pardo, G.; Monica Sabater, M.; Hummel, M.; Ferrer, A.; 598 Rodriguez-Hermosa, J.I.; Ruiz, B.; Ricart, W.; Peral, B.; Fernández-Real; J.M. miRNA expression profile of human subcutaneous adipose and during adipocyte differentiation. PLoS One. 2010;5(2):e9022.

18. Gentile, A.M.; Lhamyani, S.; Coín-Aragüez, L.; Clemente-Postigo, M.; Oliva Olivera, W.; Romero-Zerbo, S.Y.; García-Serrano, S.; García-Escobar, E.; Zayed, H.; Doblado, E.; Bermúdez-Silva, F.J.; Murri, M.; Tinahones, F.J.; El Bekay, R. miR-20b, miR-296, and Let-7f Expression in Human Adipose Tissue is Related to Obesity and Type 2 Diabetes. Obesity (Silver Spring) 2019;27(2):245-254.

19. Lhamyani, S.; Gentile, AM; Giráldez-Pérez, RM; Feijóo-Cuaresma, M; Romero-Zerbo, SY; Clemente-Postigo, M; Zayed, H.; Olivera, WO.; Bermúdez-Silva, FJ.; Salas, J.; Gómez, CL.; Hmadcha, A.; Hajji, N.; Olveira, G.; Tinahones, FJ.; El Bekay, R. miR-21 mimic blocks obesity in mice: A novel therapeutic option. Mol Ther Nucleic Acids. 2021 Jul 2;26:401-416.

20. Deiuliis J.A. MicroRNAs as regulators of metabolic disease: pathophysiologic significance and emerging role as biomarkers and therapeutics. Int J Obes (Lond). 2016 40(1):88-101.

21. Chartoumpekis D.V, Zaravinos A., Ziros P.G, Iskrenova R.P, Psyrogiannis A.I, Kyriazopoulou V.E, Habeos I.G. Differential expression of microRNAs in adipose tissue after long-term high-fat diet-induced obesity in mice. PLoS One. 2012;7(4):e34872.

22. Wang Y.T, Tsai P.C, Liao Y.C, Hsu C.Y, Juo S.H. Circulating microRNAs have a sex-specific association with metabolic syndrome. J Biomed Sci. 2013. 4;20(1):72.

23. Song, J.; Ouyang, Y.; Che, J.; Li, X.; Zhao, Y.; Yang, K.; Zhao, X.; Chen, Y.; Fan, C.; Yuan, W. Potential Value of miR-221/222 as Diagnostic, Prognostic, and Therapeutic Biomarkers for Diseases. Front Immunol. 2017; 8:56.

24. Vivacqua A, De Marco P, Belfiore A, Maggiolini M. Recent Advances on the Role of microRNAs in both Insulin Resistance and Cancer. Curr Pharm Des. 2017;23(25):3658-3666.

25. Clemente-Postigo, M.; Roca-Rodriguez, M.; Camargo, A.; Ocaña-Wilhelmi, L.; Cardona, F.; Tinahones, F.J. Lipopolysaccharide and lipopolysaccharide-binding protein levels and their relationship to early metabolic improvement after bariatric surgery. Surg Obes Relat Dis. 2015;11(4):933-9. 614 
26. Agarwal, V.; Bell, G.W.; Nam, J.W.; Bartel, D.P. Predicting effective microRNA target sites in 615 mammalian mRNAs. Elife 2015;4.

27. Friedman, R.C.; Farh, K.K.; Burge, C.B.; Bartel, D.P. Most mammalian mRNAs are conserved targets of microRNAs. Genome Res. 2009;19(1):92-105.

28. Mysore. R.; Ortega, F.J.; Latorre, J.; Ahonen, M.; Savolainen-Peltonen, H.; Fischer-Posovszky, P.; Wabitsch, M.; Olkkonen, V.M.; Fernández-Real, J.M.; Haridas, P.A.N. MicroRNA-221-3p Regulates Angiopoietin-Like 8 (ANGPTL8) Expression in Adipocytes. J Clin Endocrinol Metab. 2017;102(11):4001-4012.

29. Cortés-Garcia, J.D.; Briones-Espinoza, M.J.; Vega-Cárdenas, M.; Ruíz-Rodríguez, V.M.; Mendez-Mancilla, A.; Gómez-Otero, A.E.; Vargas Morales, J.M.; García-Hernández, M.H.; Portales-Pérez, D.P. The inflammatory state of adipose tissue is not affected by the anti-inflammatory response of the A2a-adenosine system and miR-221/PTEN. Int J Biochem Cell Biol. 2018;100: $42-48$.

30. Chistiakov, D.A.; Sobenin, I.A.; Orekhov, A.N.; Bobryshev, Y.V. Human miR-221/222 in Physiological and Atherosclerotic Vascular Remodeling. Biomed Res Int. 2015; 2015:354517.

31. Xie J., Han M., Zhang M., Deng H, Wu W. PP5 (PPP5C) is a phosphatase of Dvl2. Sci Rep. 2018 619 9;8(1):2715.

32. Zhao W., Zhang S., Wang X., Ma X., Huang B., Chen H., Chen D. ETS1 targets RYBP transcription to inhibit tumor cell proliferation. Biochem Biophys Res Commun. 2019. 12;509(3):810-816.

33. Banerjee, M.; Saxena, M. Interleukin-1 (IL-1) family of cytokines: role in type 2 diabetes. Clin Chim Acta 623 2012, 413: 1163-70.

34. Subramaniam S., Stansberg C., Cunningham C. The interleukin 1 receptor family. Dev Comp Immunol. 2004. 3;28(5):415-28.

35. Gong, W.; Li, J.; Wang, Y.; Meng, J.; Zheng, G. miR-221 promotes lens epithelial cells apoptosis through interacting with SIRT1 and E2F3. Chem Biol Interact. 2019; 306:39-46. doi: 10.1016/j.cbi.2019.03.021.

36. Shi, L.H.; Tian, L.; Wang, Y.F.; Liu, J.S.; Guo, M.F.; Liu, W. [Effects of MiR-221-Mediated Wnt/ $\beta$-Catenin Signaling Pathway on Biological Activity of Childhood Acute Lymphoblastic Leukemia Cells]. Zhongguo, Shi Yan Xue Ye Xue Za Zhi. 2019;27(5):13671373.

37. Xu, C.H.; Liu, Y.; Xiao, L.M.; Chen, L.K.; Zheng, S.Y.; Zeng, E.M.; Li, D.H.; Li, Y.P. Silencing microRNA-221/222 cluster suppresses glioblastoma angiogenesis by suppressor of cytokine signaling-3-dependent JAK/STAT pathway. J Cell Physiol. 2019;234(12):22272-22284.

38. Wang, L.; Liu, C.; Li, C.; Xue, J.; Zhao, S.; Zhan, P.; Lin, Y.; Zhang, P.; Jiang, A.; Chen, W. Effects of microRNA-221/222 on cell proliferation and apoptosis in prostate cancer cells. Gene. 2015;572(2):252-8.

39. Jia, Z. Yang, Y.; Dengyan, Z.; Chunyang, Z.; Donglei, L.; Kai, W.; Song, Z. RAP1B, a DVL2 binding protein, activates Wnt/beta-catenin signaling in esophageal squamous cell carcinoma. Gene 2017; 611:15-20.

40. Jang, S.; Park, J.S.; Jeong, H.S. Neural Differentiation of Human Adipose Tissue-Derived Stem Cells Involves Activation of the Wnt5a/JNK Signalling. Stem Cells Int. 2015; 2015:178618.

41. Barreyro, L. et al. Overexpression of IL-1 receptor accessory protein in stem and progenitor cells and outcome correlation in AML and MDS. Blood 2012; 120: 1290-1298.

42. Appari M., Channon K.M, McNeill E. Metabolic Regulation of Adipose Tissue Macrophage Function in Obesity and Diabetes. Antioxid Redox Signal. 2018 . 20;29(3):297-312.

43. Kabekkodu S.P, Shukla V, Varghese V.K, D' Souza J, Chakrabarty S, Satyamoorthy K. Clustered miRNAs and their role in biological functions and diseases. Biol Rev Camb Philos Soc. 2018. 93(4):1955-1986.

44. Karczewska-Kupczewska, M.; Stefanowicz, M.; Matulewicz, N.; Nikołajuk, A.; Strączkowski, M. Wnt Signaling Genes in Adipose Tissue and Skeletal Muscle of Humans with Different Degrees of Insulin Sensitivity. J Clin Endocrinol Metab. 2016;101(8):3079-87.

45. Zhang, Y.; Kim, M.S.; Jia, B.; Yan, J.; Zuniga-Hertz, J.P.; Han, C.; Cai, D. Hypothalamic stem cells control ageing speed partly through exosomal miRNAs. Nature 2017; 548:52-57. 


\section{Figure legends}

Figure 1. Expression profiles of the miR-221-3p/222-3p cluster in human VAT and SAT. miRNAs VAT ( $\mathrm{n}=12$ ) and SAT $(n=12)$ gene expression levels were measured by qPCR, and relative quantification of the expression levels was carried out with the comparative threshold cycle $(\mathrm{Ct})$ method. Has-miR-16 was used as an endogenous control. Data are expressed as the mean \pm SEM and were submitted to multivariate general linear models with groups of tissue (VAT vs. SAT), diabetes (NG vs. type 2 diabetic subjects) and obesity (NW vs. OB) as independent variables. $\ddagger \mathrm{p}<0.05$ for the difference in tissue group.

Figure 2. Bioinformatic analysis. A. A total of 53 validated TGs, which are common genes for miR-221-3p and miR222-3p were analyzed with PANTHER to highlight the signaling pathway in which they are involved. B. A total of 24 TGs, 7 validated and 17 non-validated, involved in adipogenesis, angiogenesis and apoptosis pathway were introduced in GeneCodis3 for enrichment analysis which highlighted 3 TGs involved in angiogenesis and apoptosis pathways. C. An interaction network was created using Cytoscape software. This network has white nodes for pathways and grey or black nodes for TGs. Edges show the miRNA involved in the interaction between TGs and their biological functions (solid: miR-221-3p and miR-222-3p; sinewave: miR-221-3p; equal dash: miR-222-3p).

Figure 3. A. mRNA expression levels of ETS1, DVL2 and IL1RAP in human VAT and SAT. Gene expression levels were measured from VAT and SAT of NG-NW, NG-OB, IR-OB and D-OB groups. The data are expressed as the mean ${ }_{ \pm}$SEM. A multivariate general model was used for statistical analysis ( $\ddagger p<0.05$ for the difference in tissue group). B.

Interaction network between miRNAs, TGs, and pathways. An interaction network was created using Cytoscape software. This network has grey nodes for miRNAs and black nodes for TGs. Edges show the pathways involved in the interaction between TGs and miRNAs (dash: apoptosis; sinewave: angiogenesis; dots: Wnt signaling pathway). 
Figure 4. Expression and Regulatory Patterns of miRNAs/mRNAs. An interaction network was created to show the expression and regulatory pattern of the miR-221-3p/222-3p cluster/ETS1, DVL2, IL1RAP. This network has green hexagons for under-expressed miRNA, red hexagons for over-expressed miRNA, green ellipse for down-regulated mRNA and red ellipse for up-regulated mRNA. 


\section{TABLES}

Table 1. Anthropometric and biochemical characteristics of the study groups.

\begin{tabular}{|c|c|c|c|c|c|}
\hline & NG-NW & D-NW & NG-OB & IR-OB & D-OB \\
\hline & $(n=7)$ & $(n=3)$ & $(n=7)$ & $(n=4)$ & $(n=7)$ \\
\hline \multirow[t]{2}{*}{ BMI $\left(\mathrm{kg} / \mathrm{m}^{2}\right)$} & $22.74 \pm 0.46$ & $25.00 \pm$ & $34.57 \pm 2.04$ & $36.02 \pm$ & $37.49 \pm 2.52^{f}$ \\
\hline & $a, b, g$ & $0.32 \mathrm{a}, \mathrm{f}$ & $\mathrm{b}$ & 2.98 & $\mathrm{~g}$ \\
\hline \multirow[t]{2}{*}{ Age (years) } & $43.43 \pm 4.40^{\mathrm{a}}$ & $64.00 \pm$ & $48.00 \pm 4.47$ & $47.5 \pm$ & $50.14 \pm 7.26$ \\
\hline & & $2.00^{a}$ & & 6.74 & \\
\hline Sex (Man/Woman) & $4 / 3$ & $1 / 2$ & $4 / 3$ & $2 / 2$ & $3 / 4$ \\
\hline \multirow[t]{2}{*}{ Glucose (mg/dL) } & $89.14 \pm 3.20$ & $153.67 \pm$ & $96.17 \pm 3.84$ & $99 \pm$ & $128.64 \pm 3.36$ \\
\hline & $\mathrm{a}, \mathrm{g}$ & 29.55 a & d & $1.47 \mathrm{e}$ & $\mathrm{d}, \mathrm{e}, \mathrm{g}$ \\
\hline \multirow[t]{2}{*}{ HOMA-IR } & $1.43 \pm 0.14 \mathrm{~g}$ & $2.60 \pm 0.52$ & $1.97 \pm 0.43^{c}$ & $5.14 \pm$ & $7.09 \pm$ \\
\hline & & f & d & $0.57^{c}$ & $1.26^{\mathrm{d}, \mathrm{f}, \mathrm{g}}$ \\
\hline \multirow[t]{2}{*}{$\begin{array}{l}\text { Cholesterol } \\
(\mathrm{mg} / \mathrm{dL})\end{array}$} & $\begin{array}{c}179.43 \pm \\
7.73\end{array}$ & $228.67 \pm$ & $171.67 \pm$ & $218.25 \pm$ & $182.14 \pm$ \\
\hline & & 31.17 & $12.37^{c}$ & $15.77 \mathrm{c}$ & 11.66 \\
\hline \multirow[t]{2}{*}{$\begin{array}{l}\text { Triglycerides } \\
\text { (mg/dL) }\end{array}$} & $75.00 \pm$ & $163.67 \pm$ & $80.86 \pm$ & $156.25 \pm$ & $175.93 \pm$ \\
\hline & $13.08^{a, g}$ & $21.88^{a}$ & $11.51^{c, d}$ & $23.79^{c}$ & $29.74^{\mathrm{d}, \mathrm{g}}$ \\
\hline \multirow[t]{2}{*}{ HDL-c (mg/dL) } & $53.14 \pm 6.11$ & $51.00 \pm$ & $49.50 \pm 5.92$ & $44.25 \pm$ & $39.36 \pm 3.91$ \\
\hline & & 3.46 & & 2.53 & \\
\hline \multirow[t]{2}{*}{ LDL-c (mg/dL) } & $111.71 \pm$ & $121.00 \pm$ & $91.74 \pm$ & $140.53 \pm$ & $118.81 \pm$ \\
\hline & 11.14 & 21.22 & $17.59^{c}$ & $13.34^{c}$ & 11.62 \\
\hline
\end{tabular}


Donors $(n=28)$ were classified according to BMI, HOMA-IR, and glycemic state. Data are expressed as the mean \pm SEM. Comparison between groups was performed by the Mann-Whitney $U$ test, if the data is not a normal (Gaussian) distribution or Student's t test, if the data is a normal (Gaussian) distribution. Different superscript letters represent statistically significant differences between groups $(\mathrm{p}<0.05)$ as follows: a: D-NW versus NG-NW; b: NG-OB versus NGNW; c: IR-OB versus NG-OB; d: D-OB versus NG-OB; e: D-OB versus IR-OB; f: D-OB versus D-NW; g: D-OB versus NG-NW. BMI: body mass index, HOMA-IR: homeostasis model assessment index; NG-NW: normoglycemic normoweight subjects; D-NW: diabetic normoweight subjects; NG-OB: normoglycemic subjects with obesity; IR-OB: normoglycemic with insulin resistance and obesity; D-OB: diabetic subjects with obesity. HDL-c: HDL-cholesterol; LDLc: LDL-cholesterol.

Table 2. Bioinformatic analysis.

\begin{tabular}{lccc}
\hline & $\begin{array}{c}\text { Validated } \\
\text { genes }\end{array}$ & $\begin{array}{c}\text { Predicted } \\
\text { genes }\end{array}$ & Total \\
\hline \multicolumn{1}{c}{ miRNA } & miRTarBase & miRWalk & \\
\hline miR-221 & 467 & 16 & 483 \\
miR-222 & 444 & 14 & 458 \\
TOTAL & 911 & 30 & 941 \\
COMMON & 53 & 14 & 67 \\
ENRICHMENT & 7 & 17 & 24 \\
ANALYSIS & & & 3 \\
SELECTED & 1 & 2 & 3 \\
\hline
\end{tabular}

miRTarBase 4.0 database was used to find validated miR-221-3p and miR-222-3p TGs. miRWalk 2.0 was used to identify predicted miR-221-3p and miR-222-3p TGs. GeneCodis3 was used for enrichment analysis. A total of 24 putative candidates were highlighted and the expression levels of 3 TGs (DVL2, ETS, IL1RAP) with the best score and specificity were analyzed in VAT and SAT samples by qPCR analysis. 


\section{FIGURE 1}

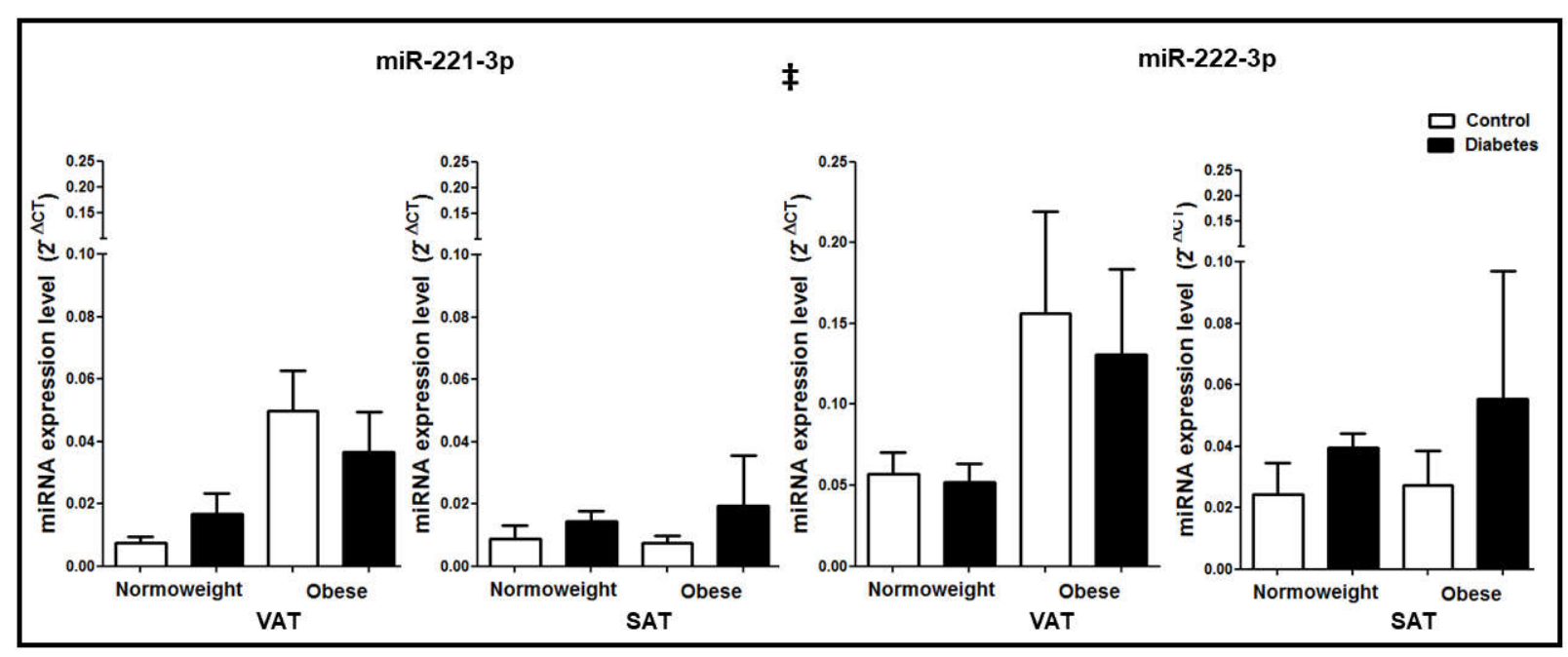

FIGURE 2
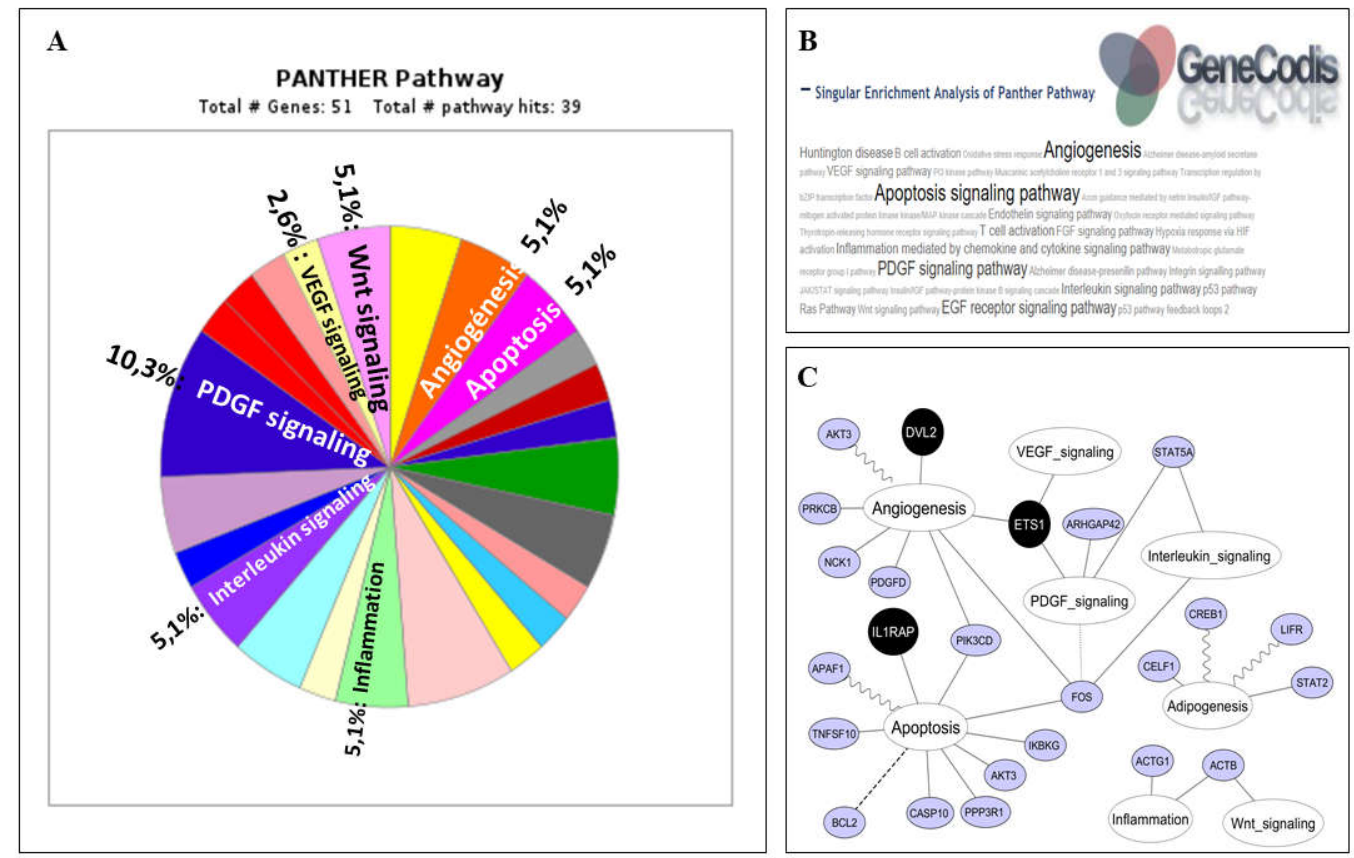


\section{FIGURE 3}

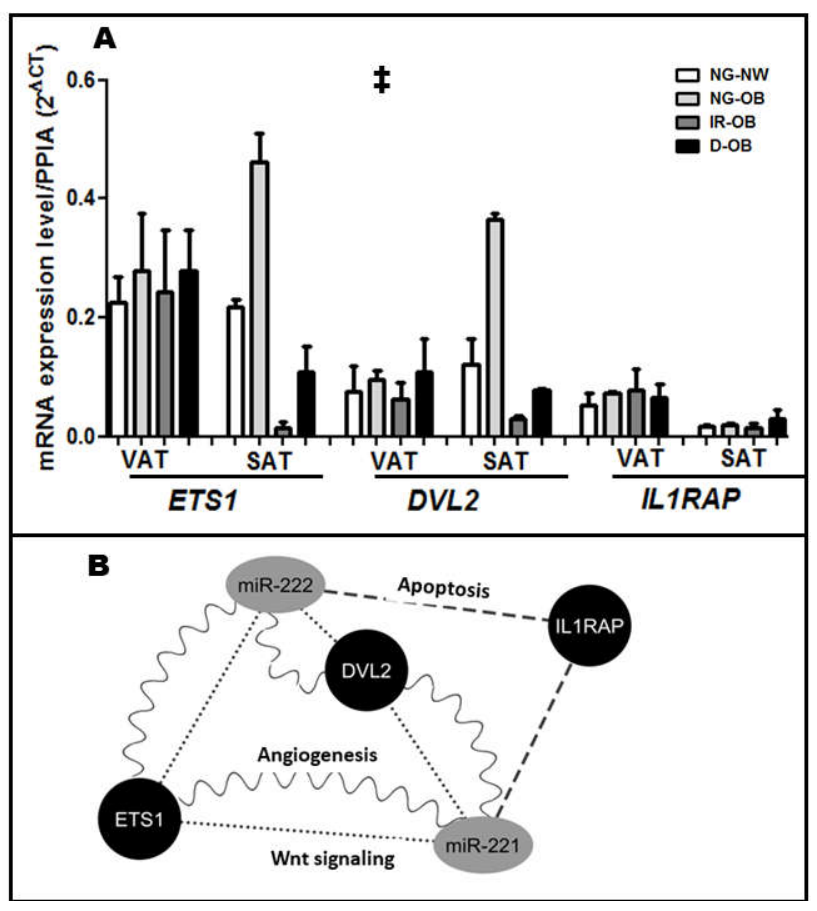

FIGURE 4

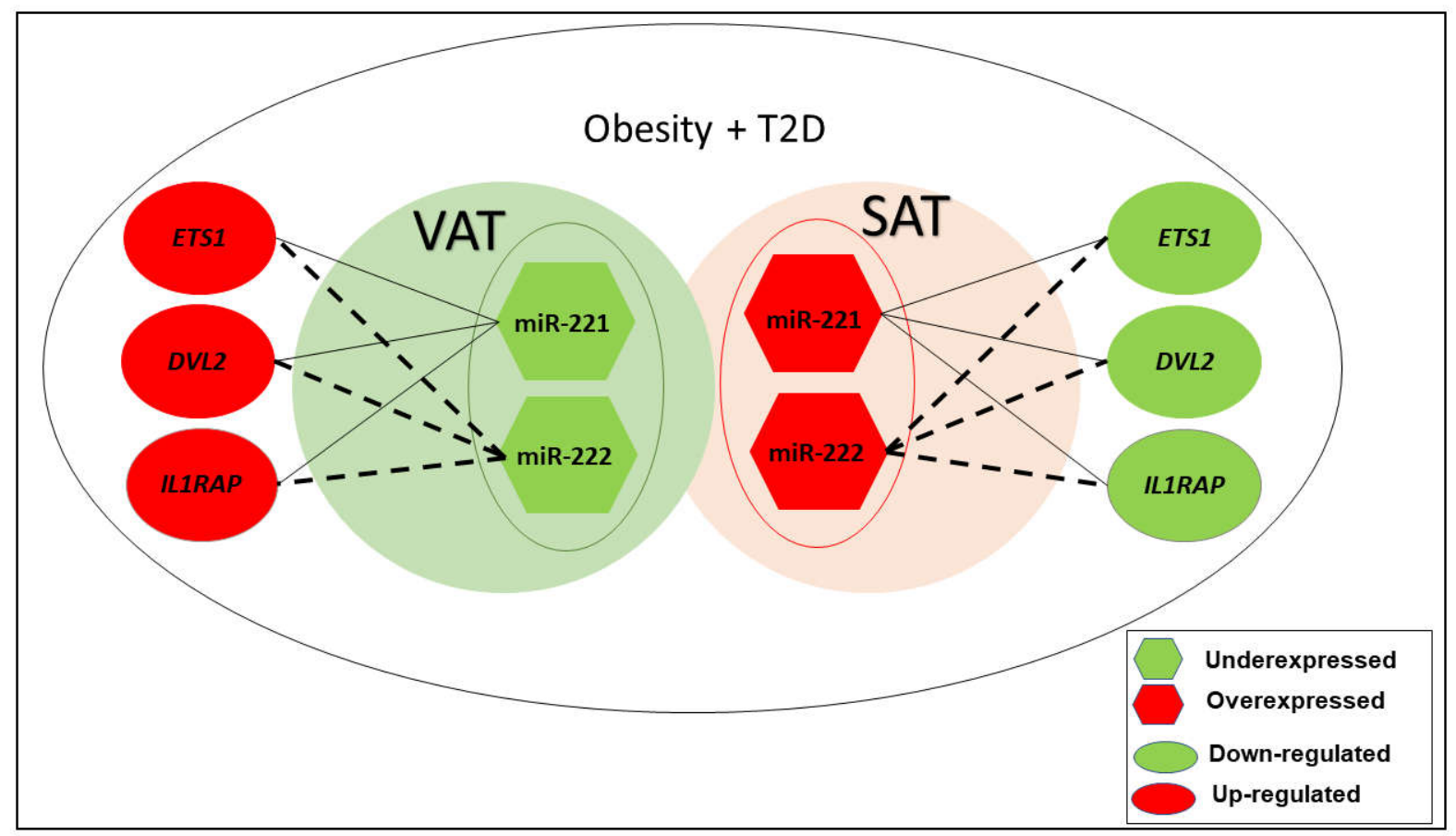

